\title{
Synchronous Gastrointestinal Stromal Tumor (GIST) of the Rectum And Jejunum: A Rare Case Report
}

Abdul Malek Bin Mohamad, Mohd Nursharmizam Baharuddin, Faisel S.A Elagili, Azmi Md Nor

Department of Surgery, International Islamic University Malaysia Medical Centre, Pahang, Malaysia.

Introduction: GIST are rare tumor and uncommon to occur in rectum where it accounts about 5\% of all GIST [1] and rarer to develop simultaneously. Several surgical options are available, large tumours with aggressive features have been commonly treated with abdominoperineal resection. This is the first reported case of synchronous GIST of the rectum and jejunum. Case report: 66 year old man who presented with rectal bleeding and altered bowel habit. Colonoscopy revealed submucosal tumor on anterior wall of the rectum. Computerized Tomography (CT) of abdomen and Magnetic Resonance Imaging (MRI) of pelvis demonstrated a mass of approximately $88 \mathrm{AP} \times 88 \mathrm{~W} \times 89 \mathrm{~mm} \mathrm{CC}$ in size on the anterior wall of the rectum at a distance of $2.3 \mathrm{~cm}$ from the anal verge, without any invasion to the prostate or evidence of metastasis, Histological examination of the biopsy sample via the rectum showed spindle cell neoplasm suggestive of GIST. An abdominoperineal resection was performed with wedge resection of small bowel lesion who discovered intraoperatively as yellow mass measuring $1.5 \mathrm{~cm}$ in its diameter involving the serosa. Histopathological examination of the sample confirmed the diagnosis of GIST of both rectal and small bowel specimen with positive CD117 on immunohistochemical assessment. Patient recovered post operatively. Discussion: Incidence of GIST as overall are about 1/100000 per year and commonly found in stomach (60-70\%), small intestine (20-25\%) but rarely occur in rectum (5\%). Because of the low incidence of rectal GISTs, cases of simultaneous development of a GIST in rectum and jejunum are scarce. Simple coincidence could be the reasonable explanation in our case. For rectal GIST, surgical procedures may be considered, including abdomino-perineal resection. Conclusion: Simultaneous GISTs in the rectum and jejunum is extremely rare. Resection is the treatment of choice, suspicious lesions discovered during operation should be biopsied or removed as GIST could appear synchronously. 